\title{
The Effectiveness of Various Attitude Indicator Display Sizes and Extended Horizon Lines On Attitude Maintenance in a Part-Task Simulation
}

\author{
J. Raymond Comstock, Jr. ${ }^{1}$, Leslie C. Jones ${ }^{2}$, and Alan T. Pope ${ }^{1}$ \\ ${ }^{1}$ NASA Langley Research Center, Hampton, VA \\ ${ }^{2}$ Virginia Tech, Blacksburg, VA
}

\begin{abstract}
Spatial disorientation (SD) is a constant contributing factor to the rate of fatal aviation accidents. SD occurs as a result of perceptual errors that can be attributed in part to the inefficient presentation of synthetic orientation cues via the attitude indicator when external visual conditions are poor. Improvements in the design of the attitude indicator may help to eliminate instrumentation as a factor in the onset of SD. The goal of the present study was to explore several display concepts that may contribute to an improved attitude display. Specifically, the effectiveness of various display sizes, some that are used in current and some that are anticipated in future attitude displays that may incorporate Synthetic Vision Systems (SVS) concepts, was assessed. In addition, a concept known as an extended horizon line or Malcolm Horizon (MH) was applied and evaluated. Paired with the MH, the novel concept of a fixed reference line representing the central horizontal plane of the aircraft was also tested. Subjects' performance on an attitude control task and secondary math workload task was measured across the various display sizes and conditions. The results, with regard to display size, confirmed the "bigger is better" concept, yielding better performance with the larger display sizes. A clear and significant improvement in attitude task performance was found with the addition of the extended horizon line. The extended or MH seemed to equalize attitude performance across display sizes, even for a central or foveal display as small as three inches in width.
\end{abstract}

\section{INTRODUCTION}

On a clear day, the view of the earth's horizon provides a pilot's peripheral visual system with constant information about the motion and position of the aircraft. Under such conditions, the maintenance of spatial orientation requires little conscious effort; the peripheral visual system is attuned to produce an intuitive sense of "which way is up," even when the pilot's attention shifts to other duties (Money, 1983). Problems arise when visual conditions outside deteriorate. Peripheral vision is the dominant and most efficient mode of spatial orientation. Therefore, clouds, night skies, and other conditions that eliminate external peripheral cues disable the pilot's optimal source for orientation information (Gillingham, 1996). With no peripheral input, the virtually effortless process of spatial orientation becomes both attention demanding and prone to error.

Poor visibility forces the pilot to exert extra effort toward actively monitoring the instrument panel's attitude indicator for synthetic orientation information in the form of pitch and bank angle. The small size of the attitude indicator relative to the natural horizon places an unnatural demand on the focal (acute) visual system for spatial orientation. Focal vision is functionally less efficient than peripheral vision in this sense, as it is specialized for the perception of fine detail, color, and object motion, not spatial orientation (Leibowitz, 1986). Focal vision spans only the central 30 degrees of the visual field; the pilot cannot focus elsewhere on the instrument panel to perform other duties if focal vision is directed toward the attitude indicator, and vice versa (Gillingham, 1996). If the pilot becomes fascinated with the attitude indicator at the expense of monitoring other instruments, a loss of situation awareness may occur. On the other hand, if the attitude indicator is ignored or misinterpreted, the lack of proper visual cues may lead the pilot to react to the compelling but erroneous vestibular and somatosensory orientation inputs that occur during flight (Gillingham, 1996). These perceptual errors lead to the potentially fatal condition known as spatial disorientation. Spatial disorientation (SD) is defined as an "erroneous sense of one's position and motion relative to the plane of the earth's surface," (Gillingham, 1996). A recent investigation, based on this definition, attributed $12.2 \%$ of Class A Air Force mishaps from 1994-1998 to SD (Neubauer, 2000). Similar statistics have been found across military, commercial, and general aviation throughout history. Despite technological advances and increased amounts of pilot training, the rate of fatal aviation accidents attributed to SD has remained relatively stable at approximately $15 \%$ for as long as accident statistics have been recorded (Stevens, 1997).

The goal of the present research was to investigate a method that may be used to reduce the risk of SD by contributing to the design of an attitude display that would more effectively present attitude information than current attitude indicators do. Various display concepts were evaluated based on the subjects' performance on an attitude control task and secondary workload task. The specific concepts tested were attitude display size, an extended horizon line based on Dr. Richard Malcolm's "Malcolm Horizon" concept, and the extended horizon with a fixed and lighted reference line.

\section{Display Size}

One purpose of this study was to evaluate the effects of attitude indicator display size on attitude control and relative workload. Several of the attitude indicator sizes of interest in this study were those of proposed Synthetic Vision Systems 
(SVS) displays, a current research area of the NASA Aviation Safety Program (AvSP). The primary goal in developing SVS is to eliminate poor visibility as a factor in aviation accidents by presenting a clear, sunny day view of photo-realistic terrain at all times, regardless of external conditions. SVS displays should enhance the presentation of attitude information by providing an easily interpretable artificial horizon that appears almost identical to a scaled down view of the real world.

Determining an optimal display size is a current challenge to the developers of SVS because future implementation of SVS depends on the feasibility of retrofitting past and current generation instrument panels that have limited amounts of available space. Retrofit instrumentation involves a trade-off between two problems: the need to conserve instrument panel space by reducing display size and the need to install instruments that are large enough to be effectively perceived by the pilot's visual system. Human perceptual issues cannot be ignored in the design of instrumentation. By testing various sizes of an attitude display in a highly controlled situation, the objective was to determine performance levels on primary attitude maintenance task, and secondary workload inducing (math addition and subtraction) tasks that corresponded to the display size.

\section{Malcolm Horizon}

Another objective of the present research was to apply a concept known as the Malcolm Horizon (MH) and evaluate its effects on attitude control and relative workload. The Malcolm Horizon is a line of light, projected onto the instrument panel, which extends the attitude indicator's artificial horizon into the pilot's periphery. The line of light, a red laser line in the present study, is presented to the left and right of the attitude display and moves with the artificial horizon relative to the earth's surface as the plane rolls and changes pitch.

The Malcolm Horizon has been compared to a large attitude indicator, although this is a slightly inaccurate description (Malcolm, 1983). Unlike the attitude indicator, the Malcolm Horizon does not have to be consciously monitored by the pilot in order to function properly. In fact, this is the basic concept behind the $\mathrm{MH}$; it was created in order to utilize the peripheral visual system for its superior ability to process orientation cues efficiently and subconsciously. When the Malcolm Horizon is applied, according to the theory, it serves to increase the pilot's ability to maintain continuous control and awareness of aircraft attitude as well as to decrease the overall workload of instrument flight (Malcolm, 1983).

As an orientation device, several characteristics of the $\mathrm{MH}$ make it a better source of attitude information than the attitude indicator alone. First, the large size of the $\mathrm{MH}$ and the fact that peripheral vision continually processes cues from the $\mathrm{MH}$ reduces the likelihood of deviations from level flight going unnoticed by the pilot (Money, 1983). Therefore, corrections can be made before unusual attitudes have the chance to occur. Another advantage is that the $\mathrm{MH}$ provides peripheral orientation cues even when vibrations from turbulence blur the retinal image of the instruments. In addition, peripheral vision continues to function properly in detecting the $\mathrm{MH}$ when high stress slows foveal instrument interpretation or provokes the coning of vision or fascination with one instrument (Money, 1983).

As a workload reduction device, the MH permits the pilot to perform better on other duties because peripheral vision detects movements of the extended MH even when the pilot's attention is directed away from the attitude indicator (Money, 1983). Since the pilot does not have to focus directly on the $\mathrm{MH}$ to gather roll and pitch information, focal vision is freed to tend to the various other instruments that must be monitored. The reduction in workload would be especially advantageous in unusual situations where workload is high and pilots are under a great deal of stress to maintain control of the aircraft (Money, 1983).

A review of the literature concerning the $\mathrm{MH}$ theory shows that an abundance of research was conducted on the topic in the early 1980's and peripheral horizon lines were even installed in several types of military aircraft, including the SR71 Black Bird. Conclusions varied; some studies found support for the $\mathrm{MH}$ and some studies found that the $\mathrm{MH}$ had no effect. The evidence of the studies overall was not great enough for the idea to be extended to commercial implementation. Speculation that the $\mathrm{MH}$ would only be beneficial with adequate pilot training was a common complaint of researchers and was one limitation in the present study since only a short period of subject exposure to the concept was possible. Problems with the actual installation and implementation of the pitch scale of the $\mathrm{MH}$ also contributed to the halt in research and some physical characteristics of the earlier $\mathrm{MH}$ implementations were not compatible with many instrument panels. These obstacles, however, do not merit discarding of the idea. There is much evidence supporting the idea of peripheral vision displays.

In the present study, it was hypothesized that the addition of an extended horizon line to the attitude display would lead to better performance on both the attitude task and the secondary workload task. This hypothesis was based on the theory behind the $\mathrm{MH}$ and the physiological evidence supporting it. An in depth discussion of the Malcolm Horizon and its advantages can be found in the NASA publication of the 1983 Peripheral Vision Horizon Display conference (Malcolm, 1983).

\section{Reference Line}

In addition to the Malcolm Horizon, the present study tested the effects of a fixed, illuminated reference line representing the horizontal plane of the aircraft. The purpose of this line was to provide a point of reference with which the Malcolm Horizon had to be aligned if level attitude was to be maintained. Expectations were that the reference line would increase the pilot's awareness of roll deviations and thus lead to better attitude stability, especially during nighttime flight when the instrument panel is not as visible as a reference to the pilot. The reference line was a novel idea in the context of Malcolm Horizon research. 


\section{METHODOLOGY}

\section{Subjects}

Twelve volunteer subjects were recruited from personnel of the crew systems branches at the NASA Langley Research Center. Eight males and four females participated, with ages ranging from 22 to 54 with a median age of 36.5 . Five subjects reported experience as Pilot-In-Command (PIC) on General Aviation aircraft with a range of PIC experience from 2 to 800 hours with a median of 55 flight hours. Five other subjects reported ground school experience but no PIC time. Two subjects reported no formal flight training experience.

\section{Attitude Display}

A Visual Basic 6 computer program was used to generate a scalable attitude indicator display. The program was run on a Toshiba Satellite Pro 6000 laptop computer and the attitude indicator display was presented to subjects on a separate, Dimension Technologies, Inc., 2015XL Virtual window LCD flat panel monitor. The attitude indicator display was square in shape for all sizes except the largest display size, which was rectangular. The display was a simplified attitude display and showed bank angle up to 45 degrees in either direction but pitch information remained fixed at a level position throughout. Table 1 shows the display sizes and visual angles for each condition tested. Size "D" refers to the size of the Primary Flight Display used on the Boeing 747-400 and 777.

Table 1. Attitude display sizes and visual angles.

\begin{tabular}{|c|c|c|c|}
\hline No. & Condition & Size $(\mathrm{cm})$ & Visual angle \\
\hline 1 & $1 "$ & $\mathrm{H}: 2.54$ x V: 2.54 & $2.39^{\circ}$ \\
\hline 2 & $3 "$ & H: 7.62 x V: 7.62 & $7.15^{\circ}$ \\
\hline 3 & Size D (3.75”) & H: 9.52 x V: 9.52 & $8.93^{\circ}$ \\
\hline 4 & $\begin{array}{l}\text { Size D+ (5.88") } \\
\text { Additional } \\
\text { Horizon } \\
\text { beyond A/S } \\
\text { and Alt. tapes }\end{array}$ & $\begin{array}{l}\text { H: } 14.92 \text { x V: } 14.92 \\
\text { No Horizon in Left } \\
\text { and Right "Tape" } \\
\text { areas of } 2.22 \mathrm{~cm} \\
\text { width each }\end{array}$ & $13.96^{\circ}$ \\
\hline 5 & $\begin{array}{l}\text { LCD panel } \\
\text { Additional } \\
\text { Horizon } \\
\text { beyond A/S } \\
\text { and Alt. tapes }\end{array}$ & $\begin{array}{l}\text { H: } 29.85 \text { x V: } 22.22 \\
\text { No Horizon in Left } \\
\text { and Right "Tape" } \\
\text { areas of } 4.44 \mathrm{~cm} \\
\text { width each }\end{array}$ & $\begin{array}{l}\text { Horizontal: } \\
27.51^{\circ} \\
\text { Vertical: } \\
20.66^{\circ}\end{array}$ \\
\hline & $\begin{array}{l}\text { Extended } \\
\text { Peripheral } \\
\text { Horizon (Laser } \\
\text { Line) and } \\
\text { Reference Line } \\
\text { (Neon Cable) }\end{array}$ & $\begin{array}{l}172.72 \mathrm{~cm} \text { total } \\
\text { width with no } \\
\text { horizon line in } \\
\text { center } 50.8 \mathrm{~cm}\end{array}$ & $\begin{array}{l}109.57^{\circ} \text { total } \\
\text { angle with } \\
\text { no horizon } \\
\text { line in center } \\
45.24^{\circ}\end{array}$ \\
\hline
\end{tabular}

Disturbances of the attitude indicator display's artificial horizon were created in the laptop software by summing two out-of-phase sine waves of differing amplitude. The correction of these disturbances was controlled by subject input through a Gravis Destroyer Xtreme Model \#10512 digital joystick connected to the laptop by a USB port. The deviation in bank angle from the level position was recorded 10 times per second.

\section{Generating the Extended Horizon}

The extended or Malcolm Horizon was generated using a laser pointer that was situated atop a tripod located behind the subject. The laser pointer utilized a "line" lens to produce a line that was divided by a $50 \%$ transmitivity beamsplitter. The resulting two red lines were projected onto a tan-colored flat surface on the left and right sides of the LCD screen, each extending from 10 to 34 inches (25.4 to $86.4 \mathrm{~cm}$ ) away from the center. Movement of the extended horizon line was accomplished using a serial line signal from the laptop to a Parallax Inc., BS2 microcontroller, which provided the pulsewidth drive signal for a Futaba FP-S148 Servo. Servo motion caused movement of the laser pointer and beamsplitter and the resulting line appeared as a peripheral extension of the central LCD displayed attitude indicator. The additional fixed reference line was constructed using Varad Corporation's Power Neon Cable, an illuminated orange strip that is commonly used as interior decoration in cars.

The attitude indicator display, extended horizon line, and reference line are depicted in Figure 1. During trials, the lights in the room were dimmed and white noise at $64 \mathrm{dBA}$ was added in the area where the subjects completed the tasks to cover over any auditory cues from the movement of the Servo.

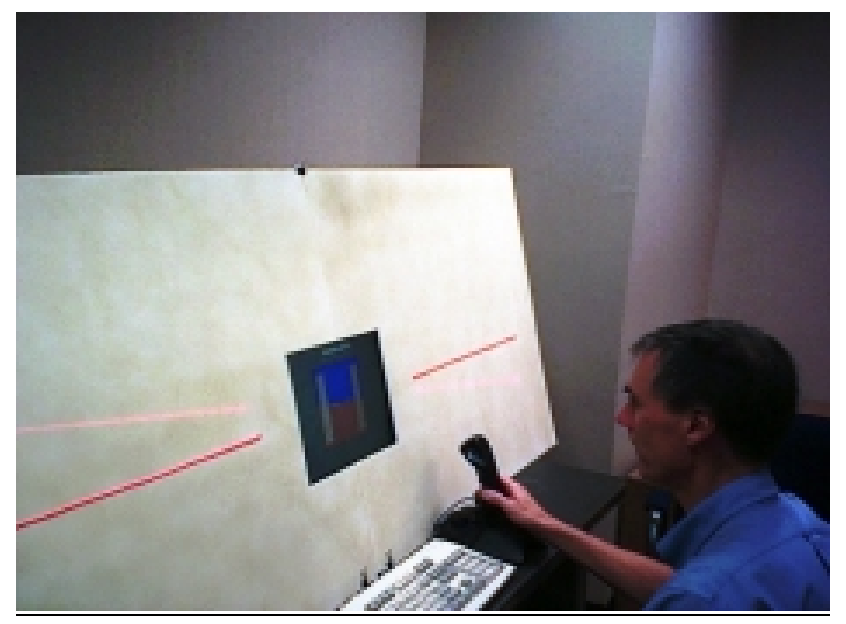

Figure 1. Digital image of the attitude display with the Malcolm Horizon and reference line. The subject uses a joystick to control attitude and a keyboard to complete the math task.

\section{Secondary Task}

While the subject maintained level attitude through the joystick control, a secondary math task was presented at the top of the screen, in order to increase workload. This required the 
subject to add two 2-digit numbers and subtract a third 2-digit number, and then use the " $\mathrm{X}$ " and " $\mathrm{C}$ " keys to increment the digits in the answer window before pressing the spacebar to register the answer. Some constraints were set in order to control the difficulty of each math problem, but the numbers were essentially generated in a random fashion. Problems were presented at the rate at which the subject answered them, but timed out if not answered within 25 seconds. Five-second delays preceded each math problem, during which the math task bar was blank. Both accuracy and completion time were dependent variables of the secondary task.

\section{Procedure}

Conditions were separated into three categories and counterbalanced across the twelve subjects. Each subject received all of the display conditions under test. The categories were attitude display only, attitude display with the $\mathrm{MH}$, and attitude display with both the $\mathrm{MH}$ and the reference line. For each category, up to five different display sizes were presented in a counterbalanced order. The subject was instructed to maintain level attitude as the primary task and complete math problems only as much as his or her attention could be directed away from the attitude display without sacrificing attitude control performance. Each subject completed two four-minute practice trials with display size D and no $\mathrm{MH}$ or reference line. During the first practice trial, the first two minutes were devoted completely to attitude control and the latter two minutes were spent concentrating only on completing the math task. The subject then coordinated both tasks in the second practice trial. There were 12 experimental conditions, and trials were each four minutes long. After each experimental trial, the NASA Task Load Index (TLX) workload rating scale was administered and the subject was prompted to estimate verbally the percentage of math problems answered correctly during that trial. An open-ended questionnaire was administered following the last trial, along with a comparison rating scale for the conditions experienced.

\section{RESULTS}

\section{Attitude task}

RMS error was the primary index of attitude task performance, and was based on the angle, in degrees, of the horizon line assessed at a rate of ten samples per second over each four-minute test interval. As shown in Figure 2, the mean RMS error was highest, indicating poorest performance for the smallest display (1"). For the conditions without the extended horizon line, performance continued to improve as display size increased. An analysis of covariance (ANCOVA) for RMS error, with secondary task (math task) completion percentage as covariate showed significant effects for Display Condition $(F(4,42)=6.227, p<.001)$. Pairwise comparisons showed that condition 1 ( 1 ") was significantly different from all the other conditions. Conditions 2, 3, and 4 did not differ significantly, however condition 5 (full screen) differed significantly from conditions 1,2 , and 3 , but not from condition 4 .

\section{Extended horizon}

The differences in RMS error for the extended horizon line are also shown in Figure 2. The ANCOVA showed that the conditions with the extended horizon line in use showed significantly better performance on the attitude maintenance task $(F(1,12)=9.234, p<.01)$. An interesting finding, as can be seen in Figure 2, is that use of the extended horizon line tended to equalize performance across the different display sizes (conditions 2-5; note that condition $1\{1$ " $\}$ was never tested with the extended horizon line). Tests of the extended horizon with and without the fixed reference line showed no significant differences in performance. This may have been a function of the reference line and the moving horizon line being too similar in color resulting in possible confusion as to which was the controllable element of the display.

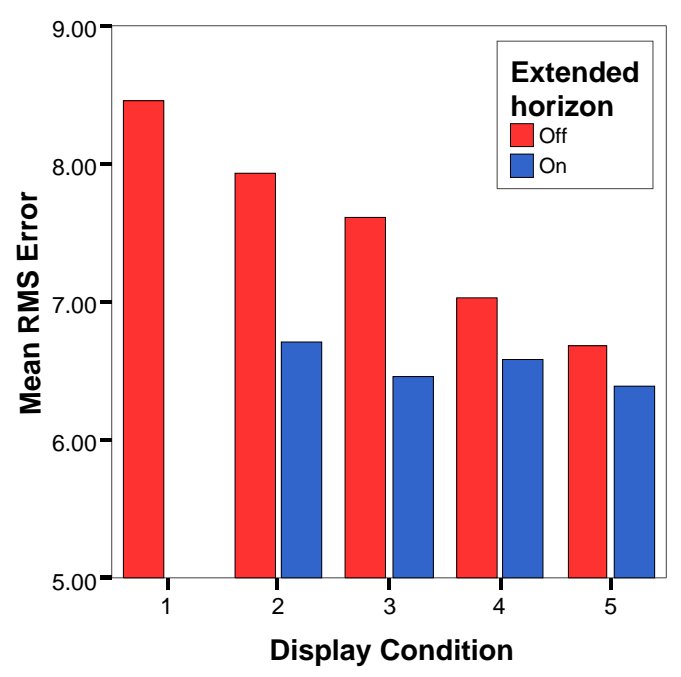

Figure 2. Plot of Attitude task RMS error by display condition, where $1=1$ "; 2 = 3"; 3 = size "D"; 4 = "D" with additional horizon beyond A/S and Alt tapes; 5 = full size of LCD display $11.75 " \mathrm{H}$.

\section{Secondary task}

Percent Math Correct (PMC) and Math Response Time (MRT) were examined as secondary task performance indices. An Analysis of Variance (ANOVA) showed that PMC did not differ significantly by display condition $(F(4,40)=1.57, p>.20)$ or for the extended horizon condition $(F(1,12)=1.44, p>.25)$. MRT showed a significant effect due to display size $(F(3,30)=2.96, p<.05)$, but an LSD post-hoc test showed this difference to be between conditions 4 (mean $=12.3 \mathrm{sec})$ and 5 (mean $=14.08 \mathrm{sec})$, and not representative of an overall display size-related trend. There was no significant difference in MRT for the extended horizon condition $(F(1,12)=.471, p>.51)$. These findings indicate that changes in performance on the 
primary task (attitude maintenance) across the display conditions did not propagate into the secondary task measures.

The number of math problems attempted over a fourminute trial ranged from 9 to 18 with a median of 14 problems attempted. The percentage of problems answered correctly ranged from $40 \%$ to $100 \%$ with a median of $83 \%$. On problems attempted, response time ranged from 8.44 to 22.08 seconds with a mean of 12.66 seconds.

\section{Subjective ratings}

NASA TLX workload ratings did not differ significantly by display or extended horizon condition. As may be expected, workload ratings did correlate positively with math response time $(\mathrm{r}=.582, p<.001)$, and negatively with both percentage of math correct $(\mathrm{r}=-.595, p<.001)$ and with the subject's estimate of math percentage correct $(r=-.600$, $p<.001)$. Subject's estimates of their math percent correct on each trial correlated highly with actual percentage correct $(\mathrm{r}=$ $0.872, p<.001)$.

\section{GENERAL DISCUSSION}

Using a part-task simulation approach, the present study was a preliminary look at the effects of attitude display size and extended horizon information on attitude task performance. With regard to display size, this study serves to confirm the "bigger is better" concept. While displays in general have increased in size over the years, optimization may not have been achieved for a given display size. For example, under test here was a display (concept 3 ) showing attitude information the size of the currently used size "D" display in which the attitude information part of the display stops inside the airspeed and altitude tape areas. With minor changes, and no change in physical hardware, additional horizon width could be built into this display, as shown in concept 4 ("D+"). In addition, an area in which attitude display size has diminished, and that has been commented on by pilots, is on the backup instruments, especially in the case of Regional Jets.

This study showed a clear and significant improvement in attitude task performance with the addition of the extended horizon line. The extended or $\mathrm{MH}$ seemed to equalize attitude performance across display sizes, even for a central or foveal display as small as three inches in width. It should also be noted that technology now permits other methods of presenting extended horizon line information perhaps through LED arrays that have directed gratings such that a separately focused left seat and right seat peripheral horizon can be achieved.

In the larger context, aviation accident reports suggest that there is a strong demand for further research concerning the prevention of SD. Instrumentation in particular is underrepresented as a topic of research, with a recent literature review of trends in SD research showing a surprisingly low number of papers written about attitude displays relative to the role that they play in SD (Previc, 2001). The present study was intended to be a preliminary investigation into some of the relevant variables for improving the attitude display with the ultimate goal of preventing instrumentation-related spatial disorientation.

An initial concern was that without a moving base simulator, the vestibular senses send signals that the body is stationary, and that these conflict with the attitude display's signals of roll changes, and according to Malcolm may decrease the extended horizon's effectiveness (Malcolm, 1983). Based on the results obtained here, the benefits of the extended horizon appeared even without motion cues.

Several limitations must be considered in interpreting the results of the present research. Of primary concern is the external validity of the attitude maintenance task used. Controlling attitude in this task only involved bank angle, and pitch information was held fixed. A logical next step would be to develop a useful extended horizon that can incorporate pitch cues as well.

\section{REFERENCES}

Gillingham, K. K., \& Previc, F. H (1996). Spatial Orientation in Flight. In R. L. DeHart (Ed.), Fundamentals of Aerospace Medicine, $2^{\text {nd }}$ Ed. (pp. 309-397). Baltimore, MD: Williams \& Wilkins.

Leibowitz, H. (1986). Recent advances in our understanding of peripheral vision and some implications. In the Proceedings of the Human Factors Society $30^{\text {th }}$ Annual Meeting (pp. 605-607). University Park, PA: Pennsylvania State University.

Malcolm, R. (1983). The Malcolm Horizon---History and future. In the R. S. Kellogg (Chair), Proceedings of the Peripheral Vision Horizon Display (PVHD) Conference (pp.12-40). NASA Conference Publication 2306 Edwards, CA: NASA.

Money, K.E. (1983). Theory underlying the peripheral vision horizon device. In the R. S. Kellogg (Chair), Proceedings of the Peripheral Vision Horizon Display (PVHD) Conference (pp. 45-56). NASA Conference Publication 2306. Edwards, CA: NASA.

Neubauer, J. (2000). Classifying spatial disorientation mishaps using different definitions. IEEE Eng Biol; 19 (2): pp. 2834.

Previc, F. H., \& Ercoline, W. R. (2001). Trends in spatial disorientation research. Aviation, Space, and Environmental Medicine, 72, no.11, p.1048-1050.

Stevens, D. E. (1997). Virtual horizon altitude warning system [A98-11101 01-54]. Proceedings of the SAFE Association Annual Symposium, $35^{\text {th }}$ (pp. 405-416). Nashville, TN. 